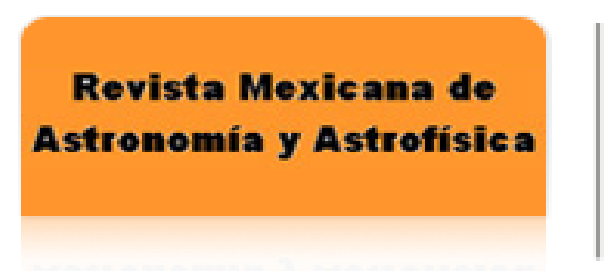

Revista Mexicana de Astronomía y Astrofísica ISSN: 0185-1101

rmaa@astroscu.unam.mx

Instituto de Astronomía

México

Richer, M. G.; López, J. A.; Lee, M. G.; Hwang, N.

Uniformity Despite Diversity: PN Expansion Velocities in the Local Group

Revista Mexicana de Astronomía y Astrofísica, vol. 28, junio, 2007, p. 107

Instituto de Astronomía

Distrito Federal, México

Available in: http://www.redalyc.org/articulo.oa?id=57102820

How to cite

Complete issue

- More information about this article

Journal's homepage in redalyc.org

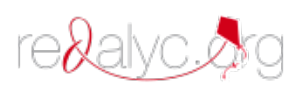

Scientific Information System Network of Scientific Journals from Latin America, the Caribbean, Spain and Portugal Non-profit academic project, developed under the open access initiative 
RevMexAA (Serie de Conferencias), 28, 107-107 (2007)

\title{
UNIFORMITY DESPITE DIVERSITY: PN EXPANSION VELOCITIES IN THE LOCAL GROUP
}

\author{
M. G. Richer, ${ }^{1}$ J. A. López, ${ }^{1}$ M. G. Lee,${ }^{2}$ and N. Hwang ${ }^{2}$
}

We have undertaken a survey of planetary nebula $(\mathrm{PN})$ expansion velocities in the [O III] $\lambda 5007$ line in Local Group galaxies visible from the OAN-SPM, Mexico using the Manchester Echelle Spectrograph (MES; Meaburn et al. 2003) on the 2.1-meter telescope. The instrumental profile has a velocity width of $11.5 \mathrm{~km} \mathrm{~s}^{-1}$ (2.7 pixel FWHM), and the nebular line profiles are always resolved.

The main results are: For the brightest of these spatially unresolved objects, asymmetries in the line profiles are clear. The mean expansion velocities (FWHM) seem remarkably uniform - despite the diversity of $\mathrm{PN}$ progenitors in different galaxies arising from very different stellar populations (Figure 1). Nevertheless, with larger samples, some differences are beginning to emerge. In M31, where we observe PNe sampling the bulge and different parts of the disk, a trend of decreasing expansion velocity with distance from the center is found. Statistically, the probability that the bulge and outer disk samples are drawn from populations with the same mean expansion velocity is only $3 \%$. The PNe in M31's outer and intermediate disk are drawn from the survey of Richer, Lee \& Hwang (2004). NGC 205 and NGC 3109 stand out for having mean $\mathrm{PN}$ expansion velocities lower than the others. The $\mathrm{O}^{2+}$ zone could sample different parts of the nebular shell in objects arising from different stellar populations, but this is unlikely to be problematic since we observe the intrinsically brightest $\mathrm{PNe}$ in $[\mathrm{O} \mathrm{III}] \lambda 5007$, where the $\mathrm{O}^{2+}$ zone encompasses the majority of the mass.

MGR and JAL gratefully acknowledge the financial support of grants from CONACyT (CONACyT 43121) and UNAM (DGAPA IN 112103).

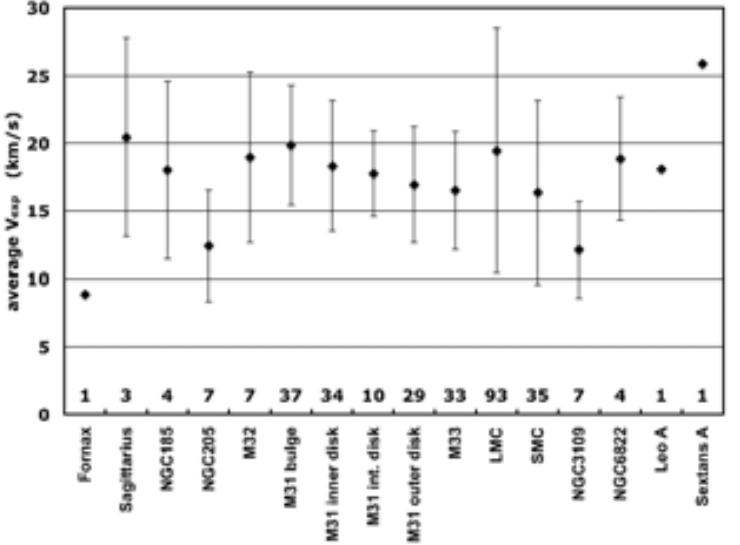

Fig. 1. For each galaxy, the point indicates the mean expansion velocity measured for its $\mathrm{PN}$ population; the error bars indicate the standard deviation of the expansion velocity distribution, and the numbers along the bottom axis indicate the number of $\mathrm{PNe}$ observed. We adopt the half-width at half-maximum as our measure of expansion velocity. Although this measure is arbitrary, most of the PNe have Gaussian line profiles at the $\mathrm{S} / \mathrm{N}$ we achieve. The data for the Magellanic Clouds were taken from the literature (Dopita et al. 1985; Dopita et al. 1988), but analyzed in the same manner as the other data.

\section{REFERENCES}

Dopita, M. A., Ford, H. C., Lawrence, C. J., \& Webster, B. L. 1985, ApJ, 296, 390

Dopita, M. A., Meatheringham, S. J., Webster, B. L., \& Ford, H. C. 1988, ApJ, 327, 639

Meaburn, J., López, J. A., Gutiérrez, L., Quirós, Murillo, J. M., Valdéz, J., \& Pedrayes, M. 2003, RevMexAA, 39,185

Richer, M. G., Lee, M. G., \& Hwang, N. 2004, J. Korean Astron. Soc., 37, 269

\footnotetext{
${ }^{1}$ Instituto de Astronomía, Universidad Nacional Autónoma de México, Apartado Postal 877, 22800 Ensenada, Baja California, México (richer@astrosen.unam.mx).

${ }^{2}$ Astronomy Program, SEES, BLD 25-1 Room 410, Seoul National University, San 56-1 Shillimdong, Gwanag-gu, Seoul 151-742, Korea.
} 